\title{
Sublingual immunotherapy for pollen allergic rhinitis: a systematic review and meta-analysis
}

\author{
Lufang Feng ${ }^{1}$, Liujiao $\mathrm{Cao}^{1}$, Peijing Yan ${ }^{2}$, Xiajing $\mathrm{Chu}^{1}$, Na Zhang ${ }^{1}$, Nan $\mathrm{Chen}^{1}$, Xingrong \\ $\mathrm{Liu}^{1}$, and kehu yang ${ }^{1}$ \\ ${ }^{1}$ Lanzhou University \\ ${ }^{2}$ Sichuan University
}

November 26, 2020

\begin{abstract}
Intro Allergic rhinitis(AR) is a common condition which can significantly impair quality of life. This study aimed to illustrate the efficacy and safety of sublingual immunotherapy (SLIT) on pollen AR patients. Methods Four electronic databases (PubMed, EMBASE, Cochrane Library, and Web of Science) were searched from their inception until September 2019. Two reviewers (FLF and CLJ) independently extracted the data. The Cochrane's Risk of Bias tool was used to assess the quality of included studies. The outcomes of study were calculated by MD or SMD with 95\%CI. A meta-analysis was performed using RevMan 5.3 software. Results In this systematic review, a total of 8 articles were included, involving 785 participants. The quality of the included studies ranged from low to moderate. The results of the meta-analysis showed that compared with placebo, a significant reduction of nasal symptoms were observed on SLIT (MD $=-0.84,95 \%$ CI $=-1.47$ to $-0.22, \mathrm{P}<0.05)$, IgE (SMD $=0.46,95 \% \mathrm{CI}=0.16$ to $0.76, \mathrm{P}<0.05)$; No significant effect on medication scores $(\mathrm{MD}=-0.41,95 \% \mathrm{CI}=-0.89$ to 0.07 , $\mathrm{P}=0.10)$. No serious adverse events were reported, and symptoms of adverse events were reported more frequently in the gastrointestinal symptoms. Conclusion SLIT can effectively relieve rhinitis symptoms and decrease the level of specific-IgE for pollen allergic rhinitis patients and the safety was verified. But due to the low quality of studies, more high-quality randomized trials are needed to provide stronger evidence of the conclusion.
\end{abstract}

\section{Hosted file}

Manuscript.doc available at https://authorea.com/users/304962/articles/495476-sublingualimmunotherapy-for-pollen-allergic-rhinitis-a-systematic-review-and-meta-analysis 


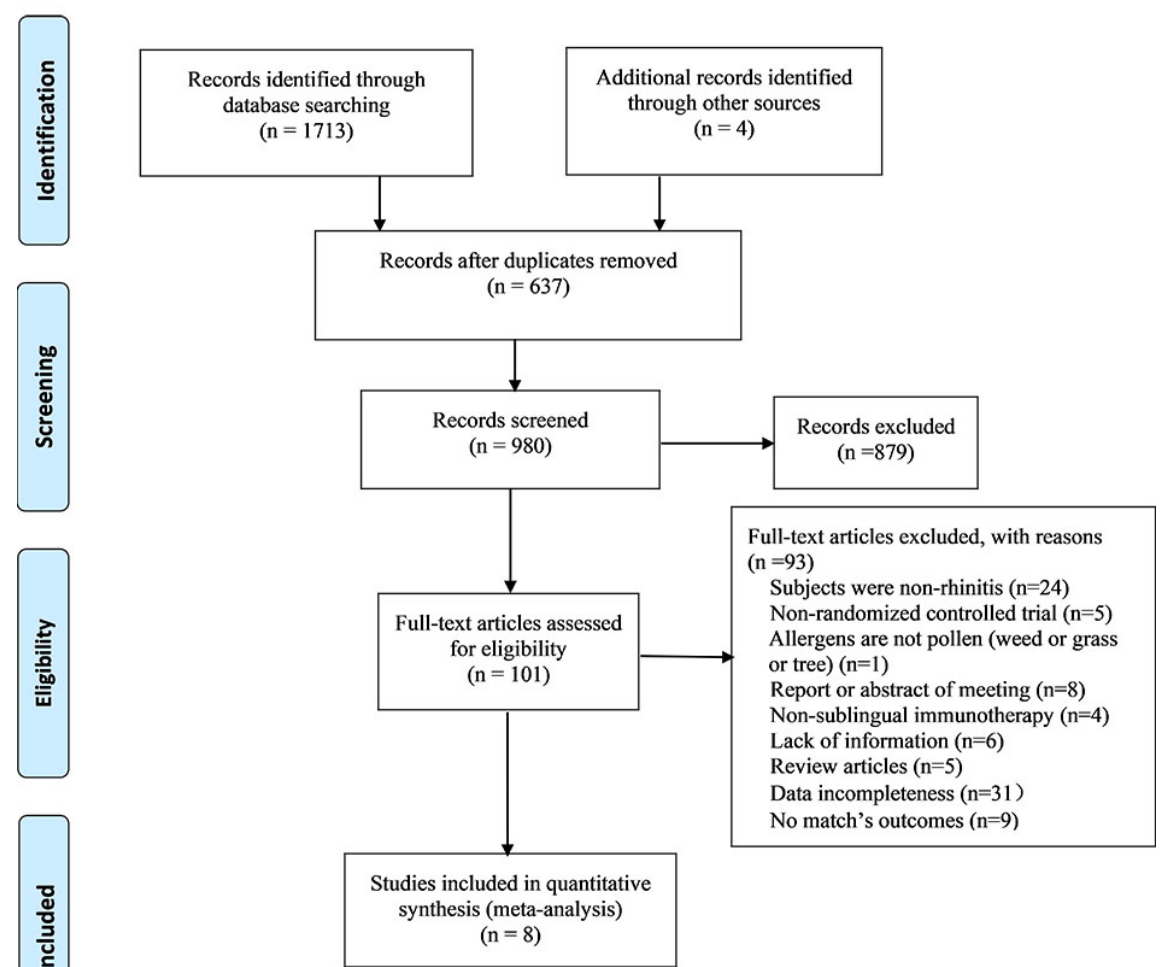




\begin{tabular}{|c|c|c|c|c|c|c|c|}
\hline & 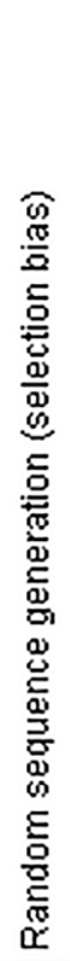 & 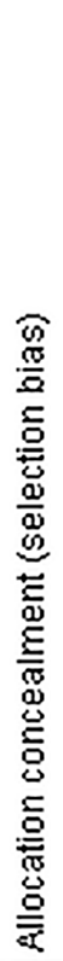 & 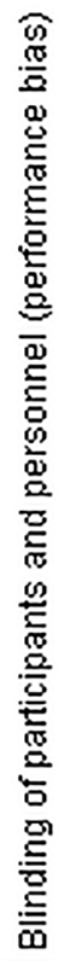 & 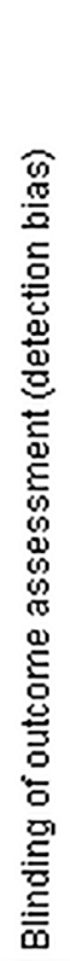 & 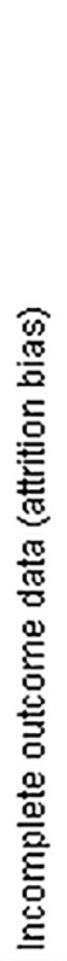 & 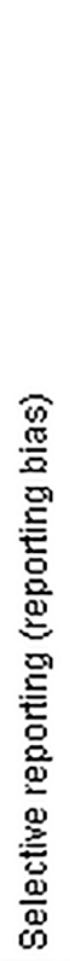 & 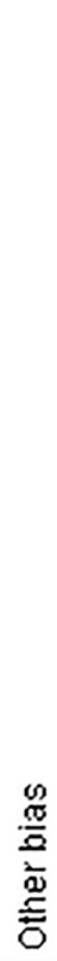 \\
\hline A. Pradalier 1999 & + & $?$ & + & $?$ & + & + & $?$ \\
\hline Akefeh Ahmadiafshar 2012 & + & $?$ & + & + & & + & ? \\
\hline Alvaro Moreno-Ancillo 2007 & + & $?$ & + & $?$ & + & + & + \\
\hline Andrzej Bozek 2014 & + & + & + & + & A & + & + \\
\hline C. André 2003 & + & $?$ & + & + & + & + & + \\
\hline Guy W.Scadding 2017 & + & $?$ & + & + & 0 & + & + \\
\hline Helen Smith MSc 2004 & + & $?$ & + & + & 2 & + & + \\
\hline Joanna Jerzynska 2016 & + & + & + & ? & 0 & + & ? \\
\hline
\end{tabular}



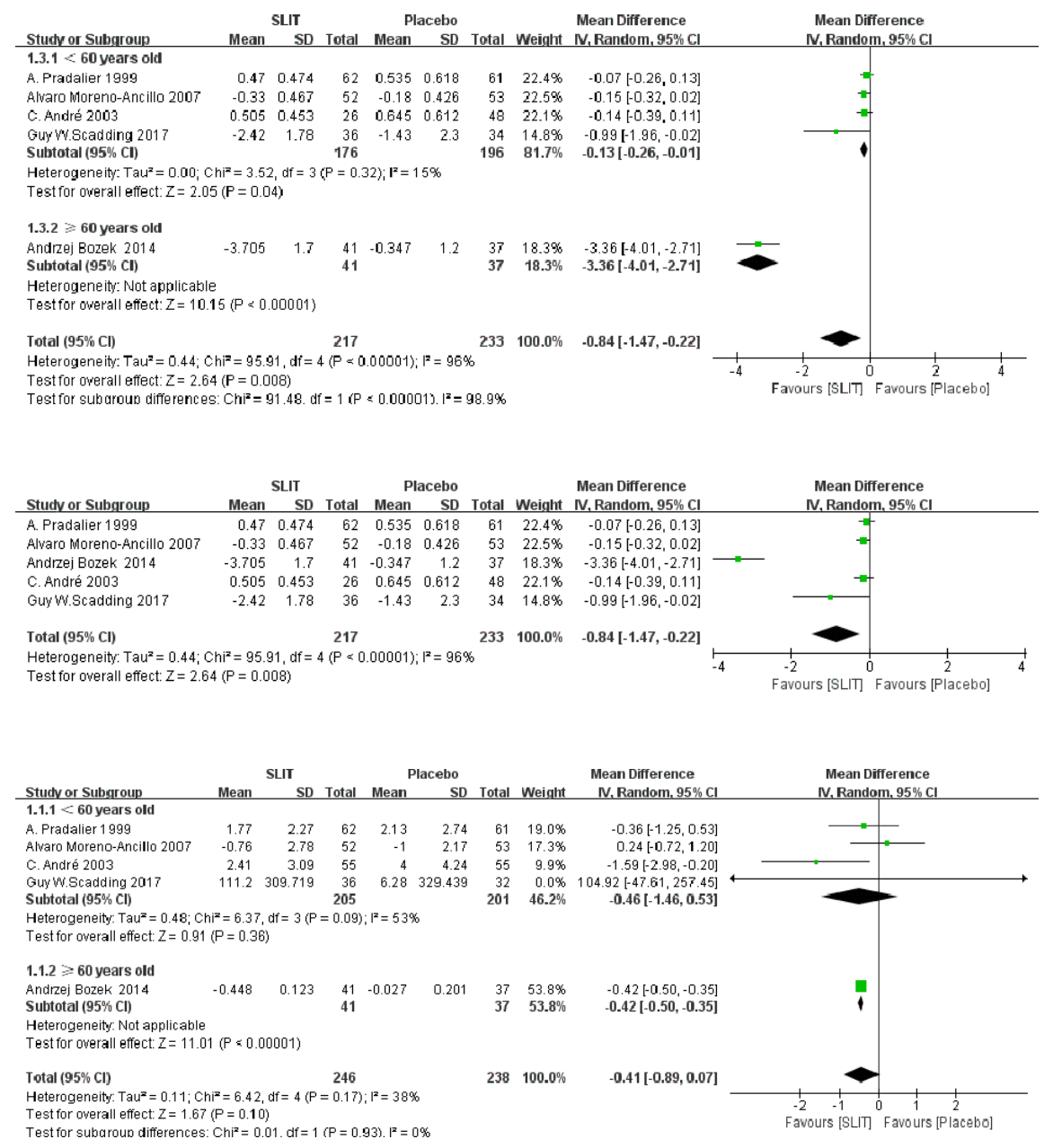